\title{
Effects of Acrylic Acid on Number Density of Polymer Particles in Emulsifier-Free Emulsion Copolymerization of Styrene and Acrylic Acid
}

\author{
Bin-Zw Yang, Leo-Wang ChEN, and Wen-Yen ChIU \\ Department of Chemical Engineering, National Taiwan University, \\ Taipei, Taiwan, Republic of China
}

(Received March 4, 1997)

\begin{abstract}
An end-chain extension model for number density of polymer particles is derived in this work. This model shows that the logarithmic value of the number density of polymer particles is proportional to the concentration of acrylic acid in aqueous solution in emulsifier-free emulsion copolymerization of styrene and acrylic acid. The end chains on polymer particles are composed of acrylic acid segments from nucleating oligomers and radicals absorbed by polymer particles. The end chains of hydrophilic segments extend into aqueous solution with surface charge at their terminals. This effect lowers the capture radical ability of polymer particles and increases the number density of polymer particles. The experimental results confirm the extension model with the content of acrylic acid ranging from 1.156 to $5.064 \mathrm{gl}^{-1}$ at $80^{\circ} \mathrm{C}$.

KEY WORDS Acrylic Acid/Styrene/Emulsifier-Free/Emulsion Copolymerization/ Number Density of Polymer Particles /
\end{abstract}

Surface charges on polymer particles are important for the properties of polymer particles. ${ }^{1-4}$ The polymer particles are stabilized by the electrical double layer repulsion force from surface charges. The repulsion force from surface charges of polymer particles and electrical charges of radicals is also important. Besides the repulsion force, the van der Waals attraction force exists between radicals and polymer particles. The capture rate constant of radicals by polymer particles depends on the interactions of these forces. The larger capture rate constant in emulsifier-free emulsion polymerization causes smaller number density of polymer particles in solution.

When the hydrophilic comonomer is added to emulsifier-free emulsion polymerization of styrene, the number density of polymer particles increases. The higher the content of the comonomer, the larger is this number density. As nucleation takes place, the hydrophilic segments from saturated oligomers are on polymer particles. When radicals are absorbed by polymer particles, the hydrophilic segments of radicals also stay on them. ${ }^{5}$ These hydrophilic segments chemically anchor on polymer particles and stabilize them. This effect causes the number density of polymer particles to increase, but varies with the solubility of the hydrophilic segment in aqueous solution. Krieger, Juang, and $\mathrm{Liu}^{6,7}$ investigated the effects of the concentration of the ionic comonomer on the particle size. They explained that the ionic comonomer provided particles with the electrostatic charge and thereby affected the primary particle formation rate, the coagulation rate, and the particle size. Ceska ${ }^{8}$ obtained a log-log plot of particle number versus weight- $\%$ itaconic acid in styrene-butadiene-itaconic acid system with slope approximating 0.6 and compared it with the Smith-Ewart theory. Chen, Chang, and $\mathrm{Lee}^{9-11}$ studied the effects of concentrations of ionic and hydrophilic comonomers on the number density of polymer particles. According to the Smith-Ewart theory in which the number density of polymer particles is proportional to the 0.6-power of emulsifier concentration in conventional emulsion polymerization of sty- rene, they suggest that $\ln N$ is proportional to the logarithmic value of the comonomer concentration. From data fitting, this number density is proportional to $[\mathrm{NaUI}],[\mathrm{NaSEM}]^{2}$, [HEMA $]^{0.17}$, and $[\mathrm{AAm}]^{0.19}$ in the systems of different comonomers, undecylenic isethionate sodium salt $(\mathrm{NaUI}),{ }^{9}$ sodium salt of 2-sulfoethyl methacrylate (NaSEM), ${ }^{9}$ 2-hydroxyethyl methacrylate (HEMA), ${ }^{10}$ and acrylamide (AAm), ${ }^{11}$ respectively. They explained the different power order of the various comonomer by the contribution of the comonomer on the particle nucleation. The different comonomers which have different reactivities and hydrophilic properties lead to different polymerization behavior in the aqueous phase and therefore the nucleation behavior and number densities of polymer particles. In this work, consideration on the electrical repulsion force between the radical and the polymer particle shows that the logarithmic value of the number density of polymer particles increases linearly with content of acrylic acid.

\section{THEORETICAL}

When nucleation and coagulation are over, the number density of polymer particles is fixed. The termination of radicals in aqueous solution can be neglected. The generation rate of radicals, $\rho_{i}$, is equal to the capture rate of radicals by polymer particles.

$$
\rho_{i}=k_{\mathrm{c}} N[\mathrm{R} \cdot]_{\mathrm{w}}
$$

where $k_{\mathrm{c}}$ is the capture rate constant of radicals by polymer particles, $N$ is the number density of polymer particles in solution, and $[R \cdot]_{\mathrm{w}}$ is the total concentration of radicals in aqueous solution. If the chain lengths of radicals in aqueous solution are the same, the diffusion coefficient, $D$, is constant for all radicals. At steady-state assumption, the flux of radicals around a polymer particle in aqueous solution, $J_{\mathrm{r}}$, is expressed as

$$
J_{\mathrm{r}}=-D \frac{\mathrm{d}[\mathrm{R} \cdot]}{\mathrm{d} r}-\frac{[\mathrm{R} \cdot]}{f} \frac{\mathrm{d} \Phi}{\mathrm{d} r}
$$


where $[\mathrm{R} \cdot]$ is the radical concentration around the polymer particle, $r$ is the radial distance from the center of the polymer particle, $\Phi$ is the potential energy between the polymer particle and the radical, and $f$ is the friction coefficient. By Einstein's equation, the expression for $f$ is

$$
f=\frac{k T}{D}
$$

where $k$ is the Boltzmann constant, and $T$ is the Kelvin temperature. Equation 2 can be rewritten as

$$
J_{\mathrm{r}}=-D \frac{\mathrm{d}[\mathrm{R} \cdot]}{\mathrm{d} r}-\frac{D}{k T}[\mathrm{R} \cdot] \frac{\mathrm{d} \Phi}{\mathrm{d} r}
$$

with boundary conditions $[\mathrm{R} \cdot]=[\mathrm{R} \cdot]_{\mathrm{w}}$ and $\Phi=0$ at $r=\infty ;[\mathrm{R} \cdot]=0$ at $r=R$, where $R$ is the radius of the polymer particle. The solution of eq 4 is

$$
J_{\mathrm{r}}=\frac{-D[\mathrm{R} \cdot]_{\mathrm{w}}}{r^{2} \int_{\mathrm{R}}^{\infty} \frac{1}{r^{2}} \exp \left(\frac{\Phi}{k T}\right) \mathrm{d} r}
$$

where the negative sign represents the absorption of radicals by polymer particles. By definition of the capture rate constant of radicals by polymer particles, $k_{\mathrm{c}}$ is expressed as

$$
k_{\mathrm{c}}=\frac{\left|J_{\mathrm{r}}\right| \cdot 4 \pi r^{2}}{[\mathrm{R} \cdot]_{\mathrm{w}}}
$$

Insertion of eq 5 into eq 6 yields

$$
k_{c}=\frac{4 \pi D}{\int_{\mathrm{R}}^{\infty} \frac{1}{r^{2}} \exp \left(\begin{array}{l}
\Phi \\
k T
\end{array}\right) \mathrm{d} r}
$$

The function $\left(1 / r^{2}\right) \exp (\Phi / k T)$ is maximum at $\Phi_{m}$ and drops off rapidly for $\Phi<\Phi_{\mathrm{m}}$. The major contribution to the integral comes from the vicinity of maximum potential energy. To neglect the higher order terms of the Taylor series expansion of $\Phi$, we obtain

$$
\Phi \approx \Phi_{\mathrm{m}}+\left.\frac{\left(r-r_{\mathrm{m}}\right)^{2}}{2}\left(\frac{\mathrm{d}^{2} \Phi}{\mathrm{d} r^{2}}\right)\right|_{m}
$$

where $r_{\mathrm{m}}$ is the distance between the center of the polymer particle and the position of the maximum potential enegy. Insertion of eq 8 into eq 7 and integration lead to

$$
k_{\mathrm{c}}=\frac{4 \pi D}{\frac{1}{r_{\mathrm{m}}^{2}} \sqrt{\frac{2 k T}{\gamma} \frac{\sqrt{\pi}}{2}}\left[1+\operatorname{erf}\left(H_{\mathrm{m}} \sqrt{\frac{\gamma}{2 k T}}\right)\right] \exp \left(\frac{\Phi_{\mathrm{m}}}{k T}\right)}
$$

where $H_{\mathrm{m}}$ is the vertical distance from the position of the maximum potential energy to the particle surface and

$$
\gamma=-\left.\frac{\mathrm{d}^{2} \Phi}{\mathrm{d} r^{2}}\right|_{m}
$$

Insertion of eq 9 into eq 1 yields

$N=\frac{1}{4 D r_{\mathrm{m}}^{2}} \frac{\rho_{i}}{[\mathrm{R} \cdot]_{\mathrm{w}}} \sqrt{\frac{k T}{2 \pi \gamma}}\left[1+\operatorname{erf}\left(H_{\mathrm{m}} \sqrt{\frac{\gamma}{2 k T}}\right)\right] \exp \left(\frac{\Phi_{\mathrm{m}}}{k T}\right)$
Taking logarithm of eq 11 leads to

$$
\begin{aligned}
\ln N= & \ln \left\{\frac{1}{4 D r_{\mathrm{m}}^{2}} \frac{\rho_{i}}{[\mathrm{R} \cdot]_{\mathrm{w}}} \sqrt{\frac{k T}{2 \pi \gamma}}\left[1+\operatorname{erf}\left(H_{\mathrm{m}} \sqrt{\frac{\gamma}{2 k T}}\right)\right]\right\} \\
& +\left(\frac{\Phi_{\mathrm{m}}}{k T}\right)
\end{aligned}
$$

The potential energy between a polymer particle and a radical is

$$
\Phi=\Phi_{\mathrm{DL}}-\Phi_{\mathrm{VDW}}
$$

where $\Phi_{\mathrm{DL}}$ and $\Phi_{\mathrm{VDW}}$ represent the potential energies of the electrical repulsion force and van der Waals force, respectively. According to Poisson's equation, the electrical potential around a polymer particle is given by

$$
\frac{1}{r^{2}} \frac{\mathrm{d}}{\mathrm{d} r}\left(r^{2} \frac{\mathrm{d} \psi_{\mathrm{DL}}}{\mathrm{d} r}\right)=-\frac{\rho^{*}}{\varepsilon_{0} \varepsilon_{\mathrm{r}}}
$$

with boundary conditions $\psi_{\mathrm{DL}}=0$ at $r=\infty ; \psi_{\mathrm{DL}}=\psi_{0}$ at $r=R$, where $\psi_{\mathrm{DL}}$ and $\rho^{*}$ are the electrical potential and the local space charge density around the polymer particle in aqueous solution, respectively, $\varepsilon_{0}$ is the permittivity of free space, $\varepsilon_{\mathrm{r}}$ is the dielectric constant, and $\psi_{0}$ is the electrical potential at the polymer particle surface. By Boltzmann's equation in electrical field, $\rho^{*}$ is related to ion concentrations as follows:

$$
\rho^{*}=\sum_{i} Z_{i} e c_{i \infty} \exp \left(\frac{-Z_{i} e \psi_{\mathrm{DL}}}{k T}\right)
$$

where $c_{i \infty}$ is the concentration of ions of $i$ in aqueous solution, $Z_{i}$ is the valency including the sign of the charge, and $e$ is the charge on electron. When $Z_{i} e \psi_{\mathrm{DL}}<k T$, eq 15 can be rewritten as

$$
\rho^{*}=\frac{-e^{2} \psi_{\mathrm{DL}}}{k T} \sum_{i} Z_{i}^{2} c_{i \infty}
$$

Substituting eq 16 into eq 14 and solving eq 14 give

$$
\psi_{\mathrm{DL}}=\frac{\psi_{0} R}{r} \exp [-\kappa(r-R)]=\frac{\psi_{0} R}{r} \exp (-\kappa H)
$$

where $H$ is the vertical distance from the radical to the particle surface, $\kappa^{-1}$ is the Debye length of a polymer particle, and

$$
\kappa^{2}=\frac{e^{2}}{\varepsilon_{0} \varepsilon_{i} k T} \sum_{i} Z_{i}^{2} c_{i \infty}
$$

When a radical possesses the electrical charge, $q$, the electrical potential energy between a polymer particle and a radical is

$$
\Phi_{\mathrm{DL}}=\psi_{\mathrm{DL}} q=\frac{\psi_{0} R q}{r} \exp (-\kappa H)
$$

In emulsifier-free emulsion copolymerization of styrene and acrylic acid, acrylic acid segments from nucleating oligomers and radicals absorbed by polymer particles are located on polymer particles and chemically anchor on them. When one end of the hydrophilic segment is free to remain in aqueous solution, the segment is called as end chain. The surface charges of polymer particles are at the terminals of end chains and extend into aqueous solution. If the extension length by a end chain, $B$, is 
defined as the vertical distance from the charge at the terminal of the end chain to the polymer particle surface, then $R$ in eq 19 can be replaced by $R+B$ and $\psi_{0}$ is the electrical potential at $r=R+B$.

$$
\Phi_{\mathrm{DL}}=\frac{\psi_{0}(R+B) q}{r} \exp [-\kappa(H-B)]
$$

when $B \ll R$, eq 20 is rewritten as

$$
\Phi_{\mathrm{DL}}=\frac{\psi_{0} R q}{r} \exp (-\kappa H) \exp (\kappa B)
$$

Let

$$
\Phi_{\mathrm{DL} 0}=\frac{\psi_{0} R q}{r} \exp (-\kappa H)
$$

Combination of eq 21 and 22 yields

$$
\Phi_{\mathrm{DL}}=\Phi_{\mathrm{DL} 0} \exp (\kappa B)
$$

When $B / \kappa^{-1} \ll 1$, then

$$
\exp (B \kappa)=1+\frac{B}{\kappa^{-1}}
$$

Insertion of eq 23 and 24 into eq 13 yields

$$
\Phi=\Phi_{\mathrm{DL} 0}\left(1+\frac{B}{\kappa^{-1}}\right)-\Phi_{\mathrm{VDW}}
$$

Insertion of eq 25 into eq 12 leads to

$$
\begin{aligned}
\ln N= & \ln \left\{\frac{1}{4 D r_{\mathrm{m}}^{2}} \frac{\rho_{i}}{[\mathrm{R} \cdot]_{w}} \sqrt{\frac{k T}{2 \pi \gamma}}\left[1+\operatorname{erf}\left(H_{\mathrm{m}} \sqrt{\frac{\gamma}{2 k T}}\right)\right]\right\} \\
& +\frac{\left(\Phi_{\mathrm{DL} 0}-\Phi_{\mathrm{VDw}}\right)_{\mathrm{m}}}{k T}+\frac{\Phi_{\mathrm{DL} 0, \mathrm{~m}}}{k T} \frac{B}{\kappa^{-1}}
\end{aligned}
$$

The higher the concentration of acrylic acid, the longer is the average length of end chains. If the variation of these average lengths in different formulas is not too large, the average extension length by end chains into aqueous solution is proportional to $F_{\mathrm{AA}} / F_{\mathrm{S}} \cdot F_{\mathrm{AA}}$ and $F_{\mathrm{S}}$ are the mole fractions of acrylic acid and styrene in the copolymer forming in aqueous solution. Let

$$
B=G\left(F_{\mathrm{AA}} / F_{\mathrm{S}}\right)
$$

where $G$ is the ratio constant. Insertion of eq 27 into eq 26 leads to

$$
\begin{aligned}
\ln N= & \ln \left\{\frac{1}{4 D r_{\mathrm{m}}^{2}} \frac{\rho_{i}}{[\mathrm{R} \cdot]_{\mathrm{w}}} \sqrt{\frac{k T}{2 \pi \gamma}}\left[1+\operatorname{erf}\left(H_{\mathrm{m}} \sqrt{\frac{\gamma}{2 k T}}\right)\right]\right\} \\
& +\frac{\left(\Phi_{\mathrm{LD} 0}-\Phi_{\mathrm{VDW}}\right)_{\mathrm{m}}}{k T}+\frac{\Phi_{\mathrm{DL} 0, \mathrm{~m}}}{k T} \frac{G}{\kappa^{-1}} \frac{F_{\mathrm{AA}}}{F_{\mathrm{S}}}
\end{aligned}
$$

When the variation of the content of acrylic acid is not large, the changes of

$$
\begin{aligned}
& \ln \left\{\frac{1}{4 D r_{\mathrm{m}}^{2}} \frac{\rho_{i}}{[\mathrm{R} \cdot]_{\mathrm{w}}} \sqrt{\frac{k T}{2 \pi \gamma}}\left[1+\operatorname{erf}\left(H_{\mathrm{m}} \sqrt{\frac{\gamma}{2 k T}}\right)\right]\right\} \\
& +\frac{\left(\Phi_{\mathrm{DLO}}-\Phi_{\mathrm{VDw}}\right)_{\mathrm{m}}}{k T}
\end{aligned}
$$

and

$$
\frac{\Phi_{\mathrm{DL} 0, \mathrm{~m}}}{k T} \frac{G}{\kappa^{-1}}
$$

are neglected in different formulas at the end of nucleation and coagulation. Then, $\ln N$ is a function of $F_{\text {AA }} / F_{\text {S }}$ only. By definition,

$$
\frac{F_{\mathrm{AA}}}{F_{\mathrm{S}}}=\frac{\mathrm{d}[\mathrm{AA}]_{\mathrm{w}}}{\mathrm{d}[\mathrm{S}]_{\mathrm{w}}}=\frac{[\mathrm{AA}]_{\mathrm{w}}\left(r_{\mathrm{a}}[\mathrm{AA}]_{\mathrm{w}}+[\mathrm{S}]_{\mathrm{w}}\right.}{[\mathrm{S}]_{\mathrm{w}}\left([\mathrm{AA}]_{\mathrm{w}}+r_{\mathrm{s}}[\mathrm{S}]_{\mathrm{w}}\right)}
$$

where $[\mathrm{AA}]_{\mathrm{w}}$ and $[\mathrm{S}]_{\mathrm{w}}$ are the concentrations of acrylic acid and styrene in aqueous solution, and $r_{\mathrm{a}}$ and $r_{\mathrm{s}}$ are the reactivity ratios of acrylic acid and styrene, respectively, and are defined by

$$
\begin{aligned}
& r_{\mathrm{a}}=\frac{k_{\mathrm{paa}}}{k_{\mathrm{pas}}} \\
& r_{\mathrm{s}}=\frac{k_{\mathrm{ps}}}{k_{\mathrm{paa}}}
\end{aligned}
$$

where $k_{\mathrm{paa}}$ and $k_{\mathrm{ps}}$ are the self-propagation rate constants of acrylic acid and styrene, and $k_{\text {pas }}$ and $k_{\text {psa }}$ are the cross-propagation rate constants between acrylic acid and styrene. When $[\mathrm{AA}]_{\mathrm{w}}$ is much larger than $r_{\mathrm{s}}[\mathrm{S}]_{\mathrm{w}}$, eq 29 is simplified as

$$
\frac{F_{\mathrm{AA}}}{F_{\mathrm{S}}}=\frac{[\mathrm{AA}]_{\mathrm{w}}}{[\mathrm{S}]_{\mathrm{w}}}\left(r_{\mathrm{a}}+\frac{[\mathrm{S}]_{\mathrm{w}}}{[\mathrm{AA}]_{\mathrm{w}}}\right)=1+\frac{r_{\mathrm{a}}}{[\mathrm{S}]_{\mathrm{w}}}[\mathrm{AA}]_{\mathrm{w}}
$$

Insertion of eq 32 into eq 28 yields

$$
\ln N=A+E[\mathrm{AA}]_{\mathrm{w}}
$$

where

$$
\begin{gathered}
A=\ln \left\{\frac{1}{4 D r_{\mathrm{m}}^{2}} \frac{\rho_{i}}{[\mathrm{R} \cdot]_{\mathrm{w}}} \sqrt{\frac{k T}{2 \pi \gamma}}\left[1+\operatorname{erf}\left(H_{\mathrm{m}} \sqrt{\frac{\gamma}{2 k T}}\right)\right]\right\} \\
+\frac{\left(\Phi_{\mathrm{DL} 0}-\Phi_{\mathrm{VDw}}\right)_{\mathrm{m}}}{k T}+\frac{G \Phi_{\mathrm{DL} 0, \mathrm{~m}}}{\kappa^{-1} k T} \\
E=\frac{G \Phi_{\mathrm{DL}, \mathrm{m}}}{\kappa^{-1} k T} \frac{r_{\mathrm{a}}}{[\mathrm{S}]_{\mathrm{w}}}
\end{gathered}
$$

\section{EXPERIMENTAL}

\section{Materials}

Styrene and acrylic acid were distilled twice under reduced pressure prior to polymerization. Potassium persulfate, hydroquinone, potassium hydrogen phthalate, sodium hydroxide, methanol, as well as hydrochloric acid solution were of analytical grade.

\section{Measurement of Conversion}

Polymerization was carried out in a one liter four-neck glass reactor. The reactor was equipped with a Teflon mixer, thermometer, condenser and rubber cork for taking samples. Adequate water was added to the reactor and heated to $80 \pm 0.5^{\circ} \mathrm{C}$ in a water bath. After styrene and acrylic acid were added, the mixture was stirred for $15 \mathrm{~min}$. When the aqueous solution containing $1.00 \mathrm{~g}$ potassium persulfate was added, the reaction began. The solution was kept at $80 \pm 0.5^{\circ} \mathrm{C}$ and stirred at $300 \pm$ $20 \mathrm{rpm}$. During the reaction, samples were taken from 
the reactor. The methanol solution containing excess hydroquinone was added to the sample to stop the reaction, and then water, methanol, and unreacted monomer were evaporated at $50-55^{\circ} \mathrm{C}$. The dried sample was weighed for conversion measurement. The formulas are shown in Table I.

\section{Measurement of Acrylic Acid Monomer in Aqueous} Solution

The sample was taken right after 3 hours of reaction. After the aqueous solution containing excess hydroquinone was added, the sample was quenched in ice bath and centrifuged under $10000 \mathrm{rpm}$. The upper clear solution was taken for gas chromatography (GC) measurement. The concentration of acrylic acid in aqueous solution was determined from a calibration curve.

\section{Measurement of Total Acid in Aqueous Solution}

Besides acrylic acid monomer, the aqueous solution may contain polyacrylic acid. Total acid including the acrylic acid monomer and the polyacrylic acid in aqueous solution was determined by titration. The preparation of samples is the same as the GC measurement. Before titration, the sodium hydroxide solution was calibrated with potassium hydrogen phthalate. The hydrochloric acid solution was calibrated with the sodium hydroxide solution. The indicator was $1 \mathrm{wt} \%$ phenolphthalein solution in ethanol. In titration, excess sodium hydroxide solution was added to a $15 \mathrm{ml}$ sample and the solution was back-titrated with $10^{-3} \mathrm{~N}$ hydrochloric acid solution.

\section{Measurement of Transmission Electron Micrographs}

The sample was taken right after 3 hours of reaction and added to aqueous solution containing excess hydroquinone. Transmission electron micrographs (TEM) were obtained using a Hitachi electron microscope H-600.

\section{Measurement of $c_{\mathrm{dist}, \mathrm{a}}$}

$c_{\text {dist,a }}$ is the distribution coefficient of acrylic acid between styrene and water. Adequate acrylic acid and $20 \mathrm{~g}$ styrene were added to $500 \mathrm{ml}$ water. Acrylic acid was added at 0.50 to $3.00 \mathrm{~g}$. The samples were stirred at $80 \pm 0.5^{\circ} \mathrm{C}$ for $30 \mathrm{~min}$ and placed in a water bath. The aqueous solution sample was measured by gas chromatography. The concentration of acrylic acid in aqueous solution was determined from a calibration curve. From mass balance, the concentration of acrylic acid in styrene phase was calculated. If $c_{\text {dist, }}$ is defined as the ratio of the acrylic acid in one liter of styrene phase and acrylic acid in one liter of aqueous solution, the average value of $c_{\text {dist, a }}$ at $80^{\circ} \mathrm{C}$ is 0.08 .

\section{RESULTS AND DISCUSSION}

\section{Effects of Acrylic Acid on Conversion}

Figure 1 shows the conversion curves for different formulas. All curves are concave upward to about $90 \%$ conversion, and then level off. The final conversion decreases from $96 \%$ to $91 \%$ as the content of acrylic acid increases from 1.156 to $5.064 \mathrm{~g}$ per liter solution. The polymerization rate increases with concentration of acrylic acid. A higher concentration of acrylic acid causes
Table I. Formulas of polymerization $\left(\right.$ at $80^{\circ} \mathrm{C}$ )

\begin{tabular}{ccccc}
\hline No. & Styrene $/ \mathrm{g}$ & Acrylic acid/g & Water $/ \mathrm{ml}$ & $\mathrm{K}_{2} \mathrm{~S}_{2} \mathrm{O}_{8} / \mathrm{g}$ \\
\hline 1 & 39.654 & 1.156 & 955 & 1.00 \\
2 & 39.700 & 3.029 & 953 & 1.00 \\
3 & 39.725 & 4.044 & 952 & 1.00 \\
4 & 39.857 & 5.064 & 951 & 1.00 \\
\hline
\end{tabular}

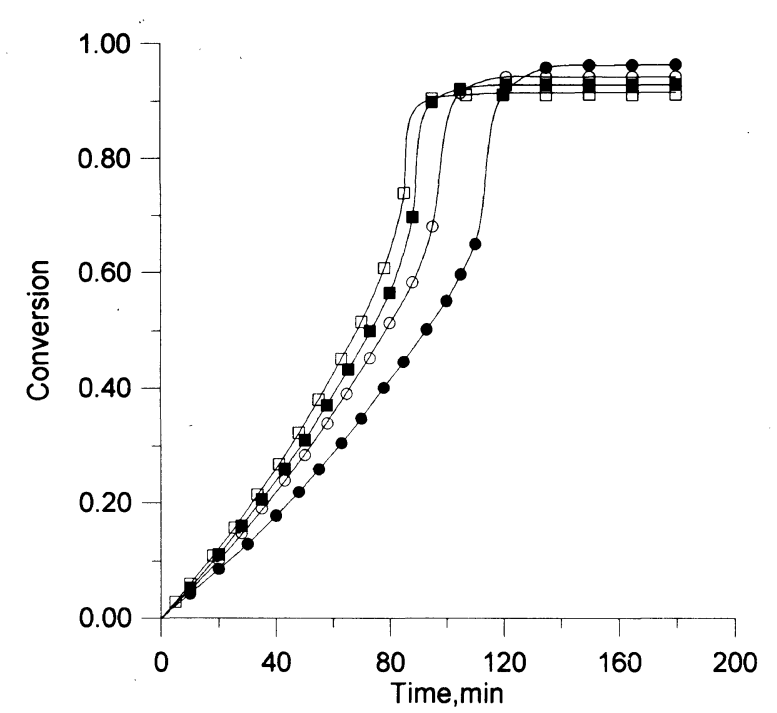

Figure 1. Conversion curves for different formulas. No. 1 , No. 2 O, No. $3 \square$, and No. $4 \square$.

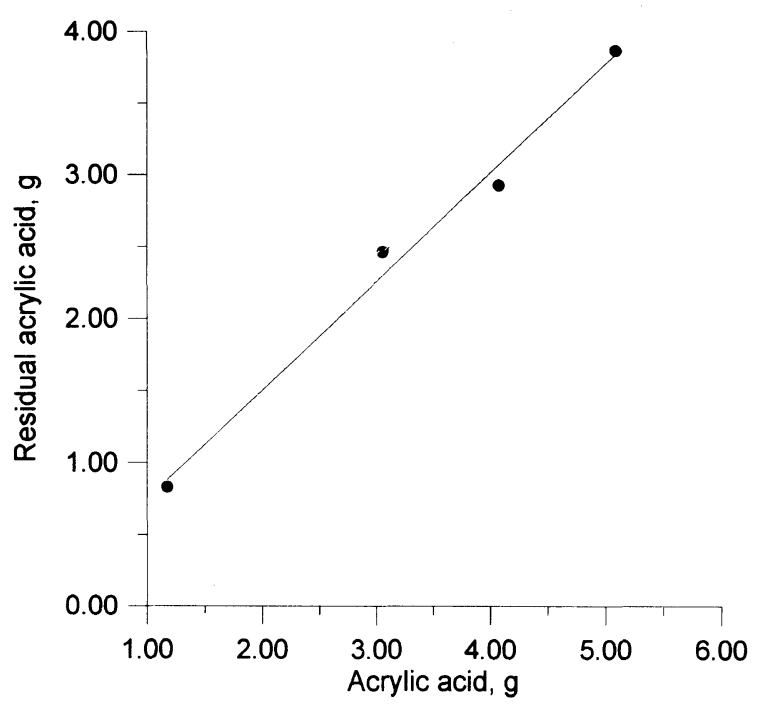

Figure 2. Residual acrylic acid in aqueous solution at 3 hours reaction as a function of content of acrylic acid.

larger number density of polymer particles and therefore results in higher polymerization rate.

Figure 2 illustrates the contents of acrylic acid in aqueous solution from GC measurement right after 3 hours of reaction. This content of acrylic acid is called the residual acrylic acid in aqueous solution. This residual acrylic acid increases with acrylic acid and is about $75 \%$. Calculation of the conversion is based on the total weight of styrene and acrylic acid. The larger the amount of residual acrylic acid, the smaller is the conversion. Thus, the final conversion decreases with increasing content of acrylic acid. 


\section{Total Acid in Aqueous Solution}

Figure 3 shows total acid in aqueous solution for

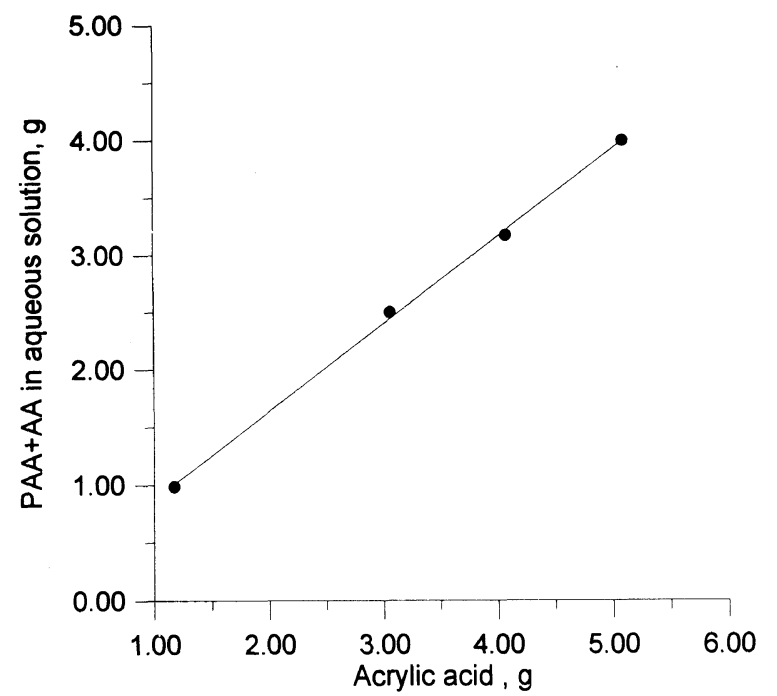

Figure 3. Total acid in aqueous solution at 3 hours reaction as a function of content of acrylic acid.

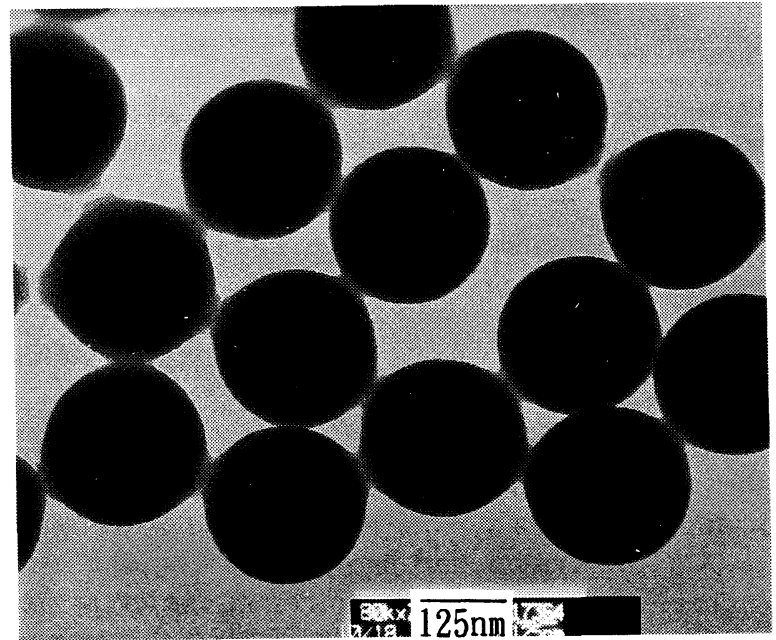

(a) No. 1

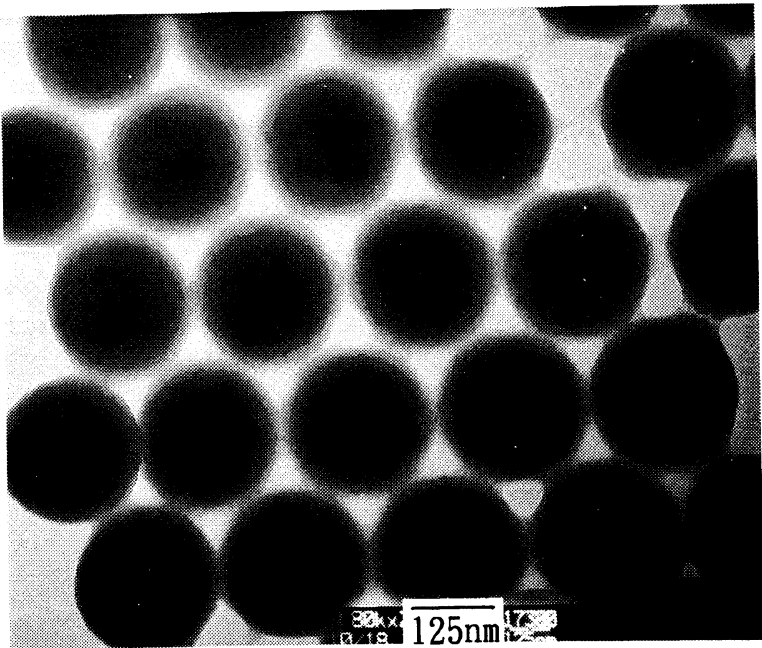

(c) No. 3 various formulas from titration right after 3 hours of reaction. A comparison of Figures 2 and 3 shows that the acid in aqueous solution is mainly monomer. The amount of polyacrylic acid is the weight difference between the amounts of total acid and residual acrylic acid in aqueous solution. The weight of poly(acrylic acid) in polymer particles is the weight difference between the amounts of acrylic acid in formula and total acid in aqueous solution. The results are listed in Table II and show that the poly(acrylic acid) is mainly in polymer particles.

Table II. Measured and calculated results at 3 hours reaction

\begin{tabular}{lcccc}
\hline Formula no. & 1 & 2 & 3 & 4 \\
\hline Conversion/\% & 96.3 & 94.1 & 92.7 & 91.1 \\
Residual AA in aqueous solution/g & 0.83 & 2.46 & 2.92 & 3.86 \\
Total acid in aqueous solution/g & 0.99 & 2.50 & 3.18 & 4.00 \\
PAA in polymer particles/g & 0.17 & 0.53 & 0.87 & 1.06 \\
PS in polymer particles/g & 39.11 & 39.68 & 39.71 & 39.84 \\
Particle diameter/nm & 246 & 227 & 217 & 208 \\
& & & &
\end{tabular}

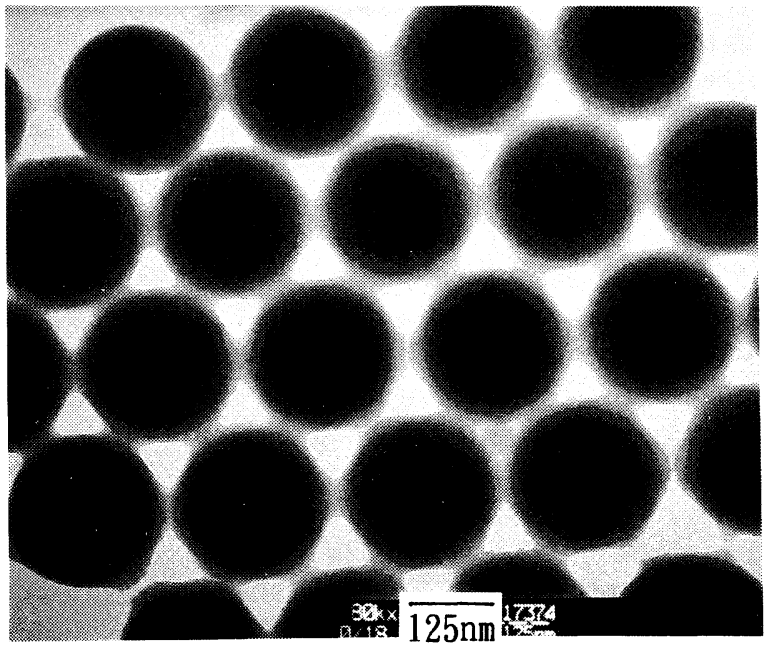

(b) No. 2

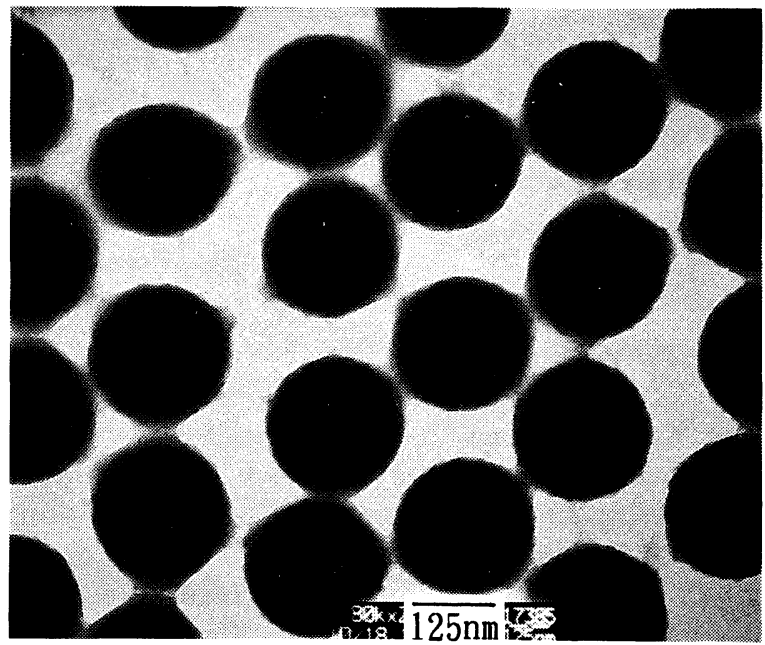

(d) No. 4

Figure 4. TEM micrographs of polymer particles for various formulas at 3 hours reaction. (a) No. 1, (b) No. 2, (c) No. 3, and (d) No. 4. 
Number Density of Polymer Particles

Figure 4 shows transmission electron micrographs for different formulas. The particle size is uniform. The radii are obtained from the transmission electron micrographs directly. The volume of one polymer particle is $4 \pi R^{3} / 3$. The total weight of polymer is calculated from the conversion and weights of styrene and acrylic acid. The weight of polystyrene is the weight difference between total weights of polymer and poly(acrylic acid) in polymer particles. The results are listed in Table II. The weight average density was used for calculation of the polymer particle volume. The density of polymer particles, $\bar{\rho}_{\mathrm{p}}$, is defined as

$$
\bar{\rho}_{\mathrm{p}}=\rho_{\mathrm{ps}} \frac{W_{\mathrm{ps}, \mathrm{p}}}{W_{\mathrm{ps}, \mathrm{p}}+W_{\mathrm{paa}, \mathrm{p}}}+\rho_{\mathrm{paa}} \frac{W_{\mathrm{paa}, \mathrm{p}}}{W_{\mathrm{ps}, \mathrm{p}}+W_{\mathrm{paa}, \mathrm{p}}}
$$

where $W_{\mathrm{ps}, \mathrm{p}}$ and $W_{\mathrm{paa}, \mathrm{p}}$ are the weights of polystyrene and poly(acrylic acid) in polymer particles, and $\rho_{\mathrm{ps}}$ and $\rho_{\text {paa }}$ are the densities of polystyrene and poly(acrylic acid) with $\rho_{\mathrm{ps}}=1.05 \mathrm{~g} \mathrm{~cm}^{-3}$ and $\rho_{\mathrm{paa}}=1.55 \mathrm{~g} \mathrm{~cm}^{-3}$, respectively. The density of poly(acrylic acid) is obtained by group contribution method. ${ }^{12}$

The total number of polymer particles $=$

$$
\frac{W_{\mathrm{ps}, \mathrm{p}}+W_{\mathrm{paa}, \mathrm{p}}}{\bar{\rho}_{\mathrm{p}}} \frac{1}{4 \pi R^{3 / 3}}
$$

The number density of polymer particles is defined as the number of polymer particles in one liter of aqueous solution. The results of calculation are listed in Table III.

In eq $33, \ln N$ is proportional to the concentration of acrylic acid in aqueous solution. Because nucleation and coagulation are short, the decrease in concentration of acrylic acid in aqueous solution can be neglected under our experimental conditions. Consequently, the concentration of acrylic acid in aqueous solution at the beginning of reaction is used in eq 33 . When $W_{\text {aa }}, W_{\mathrm{s}}$, and $W_{\mathrm{w}}$ represent the weights of acrylic acid, styrene phase, and aqueous solution, respectively, the amount of acrylic acid in aqueous solution, $W_{\mathbf{a a}, \mathbf{w}}$, is

$$
W_{\mathrm{aa}, \mathrm{w}}=\frac{W_{\mathrm{aa}}}{1+c_{\mathrm{dist}, \mathrm{a}} \frac{W_{\mathrm{s}} / \rho_{\mathrm{s}}}{W_{\mathrm{w}} / \rho_{\mathrm{w}}}}
$$

where $\rho_{\mathrm{s}}$ and $\rho_{\mathrm{w}}$ are the densities of styrene phase and aqueous solution, respectively. The concentration of acrylic acid in aqueous solution is

$$
[\mathrm{AA}]_{\mathrm{w}}=\frac{W_{\mathrm{aa}, \mathrm{w}} / M_{\mathrm{aa}}}{W_{\mathrm{w}} / \rho_{\mathrm{w}}}=\frac{W_{\mathrm{aa}} / M_{\mathrm{aa}}}{W_{\mathrm{w}} / \rho_{\mathrm{w}}+c_{\mathrm{dist}, \mathrm{s}} W_{\mathrm{s}} / \rho_{\mathrm{s}}}
$$

where $M_{\mathrm{aa}}$ is the molecular weight of acrylic acid. [AA] $]_{\mathrm{w}}$ for various formulas are listed in Table III.

Figure 5 demonstrates that the logarithmic value of the number density of polymer particles is proportional to the concentration of acrylic acid in aqueous solution. If the unit of the concentration of acrylic acid is moles in one liter of aqueous solution, the correlation is

$$
\ln N=36.00+9.44[\mathrm{AA}]_{\mathrm{w}}
$$

This result is consistent with eq 33. Although $A$ and $E$ in eq 34 and 35 are complicated functions, their values
Table III. Number density of polymer particles and concentration of acrylic acid

\begin{tabular}{lcccc}
\hline \multicolumn{1}{c}{ Formula no. } & 1 & 2 & 3 & 4 \\
\hline$N$ (particles $/$ liter $)$ & $5.04 \times 10^{15}$ & $6.48 \times 10^{15}$ & $7.50 \times 10^{15}$ & $8.57 \times 10^{15}$ \\
{$[\mathrm{AAA}]_{\mathrm{w}} / \mathrm{moll}^{-1}$} & $1.67 \times 10^{-2}$ & $4.38 \times 10^{-2}$ & $5.85 \times 10^{-2}$ & $7.33 \times 10^{-2}$
\end{tabular}

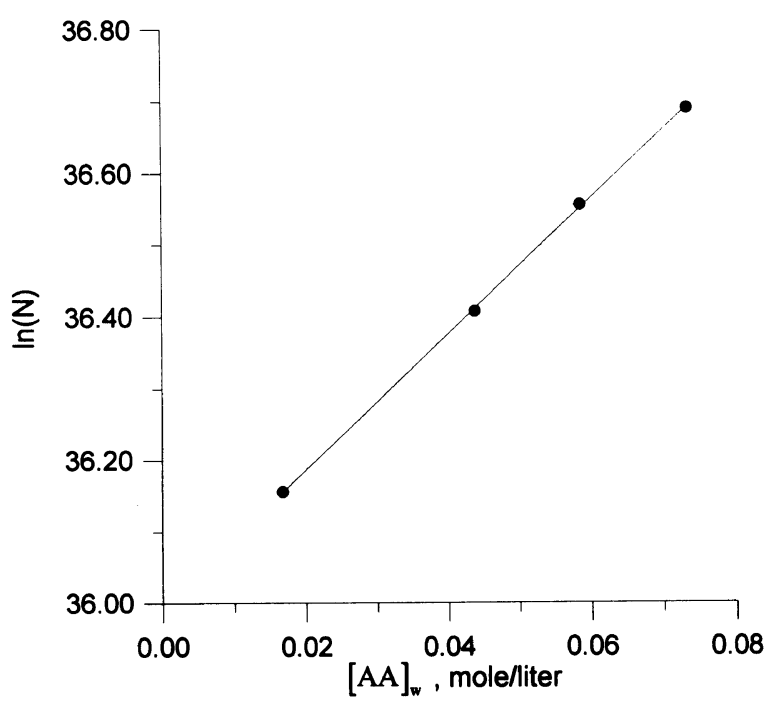

Figure 5. Logarithmic value of number density of polymer particles versus concentration of acrylic acid in aqueous solution.

can be obtained by data fitting. The results show that $A$ and $E$ are constants.

\section{CONCLUSIONS}

The hydrophilic segments on polymer particles lessen the capture radical ability of polymer particles and stabilize particles in emulsifier-free emulsion copolymerization of styrene and hydrophilic comonomer. The higher the comonomer concentration, the larger are these effects. Thus, the number density of polymer particles increases with content of comonomer.

Many experiments suggest that $\ln N$ is proportional to the logarithmic value of the hydrophilic comonomer concentration by data fitting. ${ }^{8-11}$ However, the end-chain extension model in this work shows that $\ln N$ is proportional to the concentration of acrylic acid in aqueous solution under suitable conditions in emulsifierfree emulsion copolymerization of styrene and acrylic acid. The experimental results confirm this model to be valid.

Besides the interactions of the electrical repulsion force and the van der Waals attraction force, this model is mainly based on the extension effect of end chains in aqueous solution. The nucleation rate is affected by extension effects.

\section{REFERENCES}

1. A. R. Goodall, M. C. Wilkison, and M. C. Hearn, J. Polym. Sci., Polym. Chem. Ed., 15, 2193 (1977).

2. A. R. Goodall, J. Hearn, and M. Wilkison, Br. Polym. J., 10, 141 (1978). 
3. J. W. Goodwin, R. H. Ottewill, and R. Pelton, Colloid Polym. Sci., 257, 61 (1979).

4. D. Munro, A. R. Goodall, M. C. Wilkison, K. Randle, and J. Hearn, J. Colloid Interface Sci., 68, 1 (1979).

5. S. Wang and G. W. Poehlein, J. Appl. Polym. Sci., 50, 2173 (1993).

6. M. S. Juang and I. M. Krieger, J. Polym. Sci., Polym. Chem. Ed., 14, 2089 (1976)

7. L. J. Liu and I. M. Krieger, J. Polym. Sci., Polym. Chem. Ed.,
19, 3013 (1981)

8. G. W. Ceska, J. Appl. Polym. Sci., 18, 2493 (1974).

9. S.-A. Chen and H.-S. Chang, J. Polym. Sci., Polym. Chem. Ed., 23, 2615 (1985).

10. S.-A. Chen and H.-S. Chang, J. Polym. Sci., Polym. Chem. Ed., 28, 2547 (1990)

11. S.-A. Chen and S.-T. Lee, Macromolecules, 24, 3340 (1991).

12. D. W. Van Krevelen and P. J. Hoftyzer, "Properties of Polymer," 2nd ed, Elsevier, New York, N.Y., 1976. 